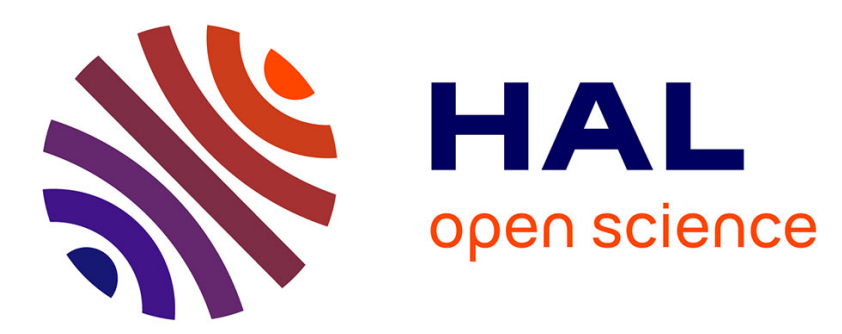

\title{
Enabling the control of a new degree of freedom by using anisotropic material on a 6-DOF parallel soft robot
}

\author{
Félix Vanneste, Olivier Goury, Christian Duriez
}

\section{To cite this version:}

Félix Vanneste, Olivier Goury, Christian Duriez. Enabling the control of a new degree of freedom by using anisotropic material on a 6-DOF parallel soft robot. Robosoft 2021, Apr 2021, Yale / Virtual, United States. hal-03194087

\section{HAL Id: hal-03194087 \\ https://hal.inria.fr/hal-03194087}

Submitted on 9 Apr 2021

HAL is a multi-disciplinary open access archive for the deposit and dissemination of scientific research documents, whether they are published or not. The documents may come from teaching and research institutions in France or abroad, or from public or private research centers.
L'archive ouverte pluridisciplinaire HAL, est destinée au dépôt et à la diffusion de documents scientifiques de niveau recherche, publiés ou non, émanant des établissements d'enseignement et de recherche français ou étrangers, des laboratoires publics ou privés. 


\title{
Enabling the control of a new degree of freedom by using anisotropic material on a 6-DOF parallel soft robot
}

\author{
Félix Vanneste*, Olivier Goury*, Christian Duriez*
}

\begin{abstract}
In this paper, we design in simulation and build a parallel soft robot with a 6 degrees of freedom (DOF) endeffector. We show that by using a 3D-printed meso-structured material which displays an anisotropic behaviour, we can modify the kinematics of the structure in order to control one additional DOF which is not possible to achieve using a standard isotropic and homogeneous material like silicone. The behaviour of the robot is simulated using numerical homogenization and the finite element method (FEM), which runs in real-time and can be used for control. We finally show that the parallel soft robot we have built is controllable in open loop thanks to the use of inverse simulation. We demonstrate its maneuverability by guiding a marble in a maze game.
\end{abstract}

\section{INTRODUCTION}

The study of the kinematics of soft robots is an exciting topic. What is fundamentally new compared to rigid robots is that the properties of the material used to build the soft robot impact the kinematics of the robot. Indeed, since the robot motion is generated by deformation, the robot will tend to move in the directions of least rigidity (or of larger compliance). Material properties become therefore a new playground for the soft robot designers. In [1], for example, the pneumatic actuation is constrained by a layer of inextensible material under the robot. In [2], a composite approach is used with an inextensible wire placed along the pneumatic cavity to constrain its deformation in one direction. In [3], a cable compresses the material at the base of a tentacle to stiffen it. And many other examples exist in the literature of soft robot actuators.

Instead of working only with actuators, we can also work on directly constraining the structure itself in order to favor some movement over others and even create new behavior. For that it seems important to better control the stiffness/compliance directions of soft robots when designing them and even ideally to program their structures with heterogeneities and anisotropies to obtain a desired behavior.

We believe that in the coming years, the community will be able to go far beyond the often-used design of isotropic, homogeneous silicon-based soft robots. The context is favorable: with the growing interest in 3D printing, some manufacturing techniques that are very simple to implement, already allow to play with material properties. In particular metamaterials can be used. The metamaterial properties are obtained by a particular micro-structuring or by the use of composite materials. They are mainly used for optic, thermal or acoustic [4] applications, but in this study we make use of them for their mechanical properties.

\footnotetext{
* University of Lille, Inria, CNRS, Centrale Lille, UMR 9189 CRIStAL, F-59000 Lille, France (DEFROST team)
}

As defined in [5] mechanical metamaterials succeed by careful design of its building blocks and by exploiting motion, deformations, stresses and mechanical energy in achieving any combination of linear elastic coefficients that is not forbidden by thermodynamics.

Regarding fabrication, mechanical metamaterials have been used already to design soft robots with various kinds of approaches such as Origami, bi-stable or auxetic structures [6. 7, 8, 9]. In this work, we use a specific type of mechanical metamaterial already introduced in our previous paper [10].

When using metamaterials or other anisotropic materials to build a soft robot, some numerical tools such as finite element analysis can help to predict its behavior. But today, these numerical techniques and the use of such parametric metamaterials are not yet integrated in a toolbox for robotics, and in particular we lack the tools for analyzing the consequences on the kinematics of the robot. The work proposed in this paper seeks to go in this direction based on the example of a parallel robot with only 5 DOF when it is made of silicone and 6 DOF when it is made of an anisotropic material.

\section{Contributions}

This paper builds on our previous work published in [10] but the new case study is of a 6 DOF parallel robot made by assembling two previously presented 3 DOF robots. We show new tools that can be used to control the 6 DOF of the end-effector independently, which is not achievable with conventional soft material. In details, the contributions are the following:

- We show we are able to control a new degree of freedom by using an anisotropic material on a parallel soft robot.

- We model and simulate it using FEM, allowing to test different configurations and making an initial assessment of the workspace.

- We finally show that the method is compatible with realtime inverse modeling, which we can use to control the real prototype in open-loop.

\section{DESIGN OF THE ROBOT}

For this study, we design a 6 DOF parallel soft robot by combining two existing 3 DOF parallel soft robots (named tripod robots). Each tripod is controlled by 3 servomotors. The 3 motors have the action of bending a soft sheet and moving it up and down.

In a previous work we have already used one of this tripod robot to show that we were able to create new kinematic by changing the soft sheet composition and organisation from 


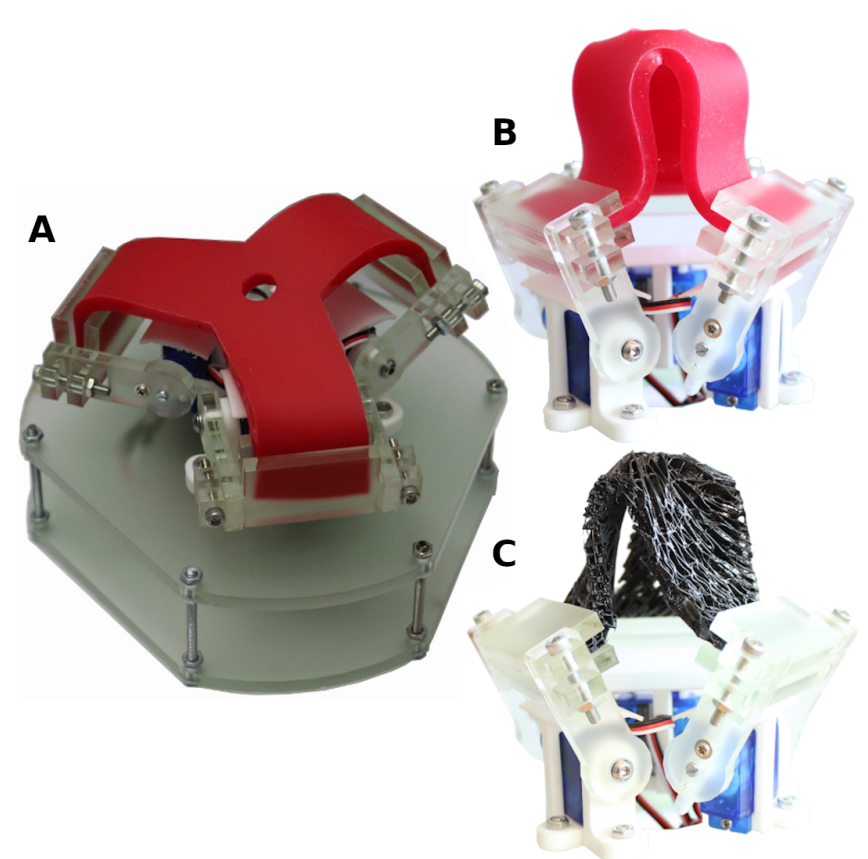

Fig. 1: A Tripod robot (A) composed of a soft silicone piece and actuated by three servomotors. In a previous work we showed that by changing the flexible sheet from silicone (B) to meso-structured material (C) we can change the kinematics and create a torsion movement.

isotropic silicone to a transverse isotropic soft foam. We were able to create a torsion as a new movement, but this torsion was coupled with a translation along the same axis. Fig. 1 show the tripod robot and the results we have obtained.

Intuitively, by putting two of them face to face and connecting them by a rigid body, we can constrain the translation allowing the system to have the sixth independent DOF. Fig. 2 show the final real setup that will be presented later. All the following experimentations will follow this configuration.

To create the anisotropy in the soft sheets, we use the procedure introduced in [11] that allow to 3D print Voronoi foams with anisotropic stiffness criteria. Fig. 3 shows That we are able to match precisely the fiber orientation of our simulated part (B) with the 3D printed foam (A). We can see that a global orientation was "programmed" into the structure in order to create some rotation when actuated.We use this flexible sheet configuration for the setup presented above. By putting two of them face to face the global system is therefore symmetric which enable a pure rotation along the axis of the rigid link when all motors moves along the same direction. Fig. 4 presents the setup in simulation and shows this rotation.

As explained above, the spiral pattern of the flexible sheets are mirrored in order to have the same behavior but if they were inversed, to have the rotations presented in Fig3, one tripod would need to "push" while the other would "pull" and vice versa to have the rotation in the other direction. We

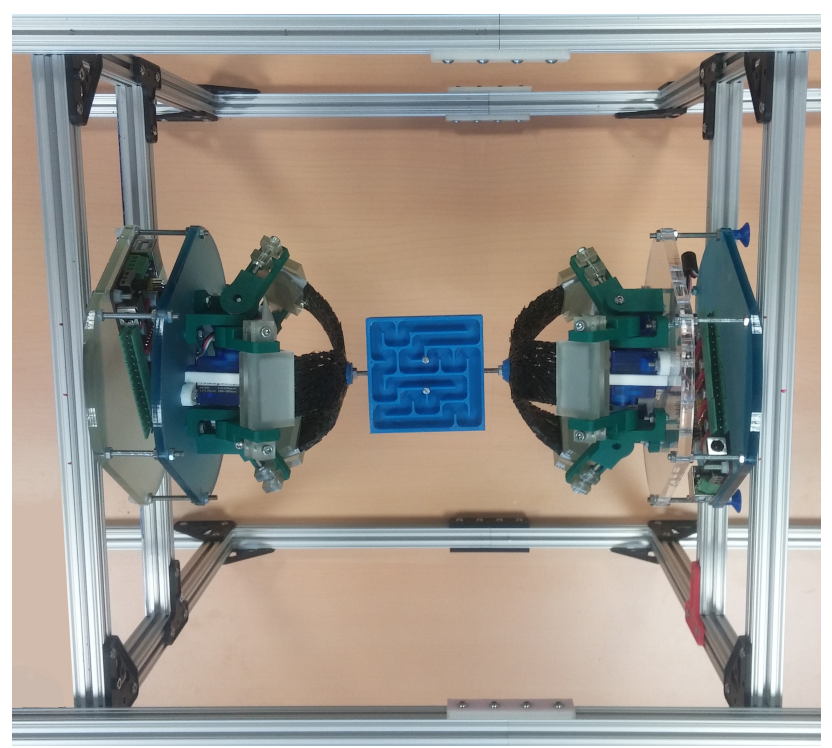

Fig. 2: View from above of the real validation setup: the 2 tripods are facing each other and linked with a rigid rod. Additionally we attached on the middle of this link a maze that will be used to visualize more efficiently the controlled movement of the end effector of this system (ie: the center of the rigid link).

choose the first option because it allows a more independent control of the DOF which we lose with the second option due to side translations.

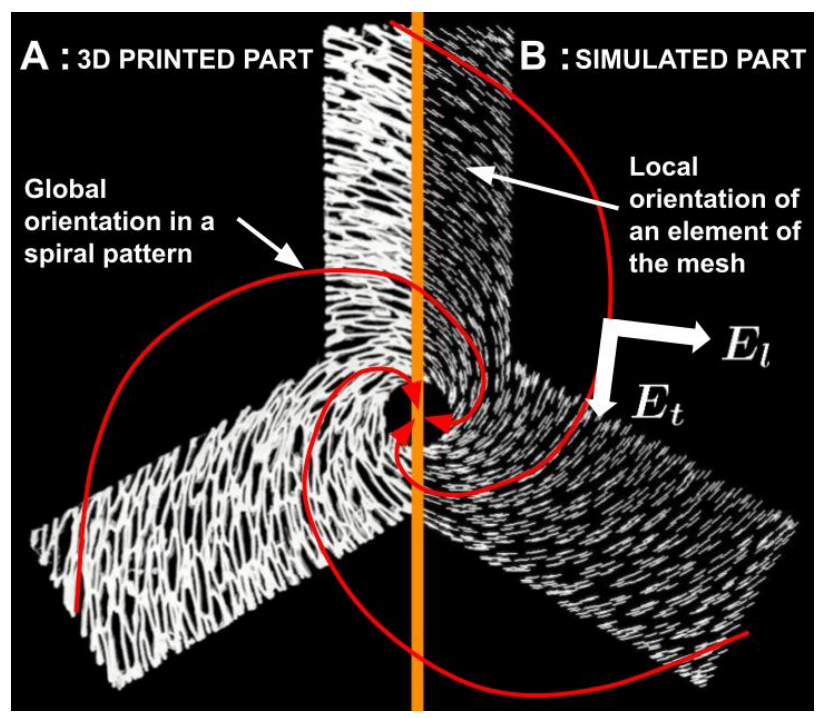

Fig. 3: View from above of a flattened flexible sheet with a comparison between the simulated part (B) where each element as a specific orientation allowing to have a global spiral pattern and the 3D printed real part (A). We keep $E_{t}$ and $E_{l}$ constant for the whole structure, only the orientation differ. 


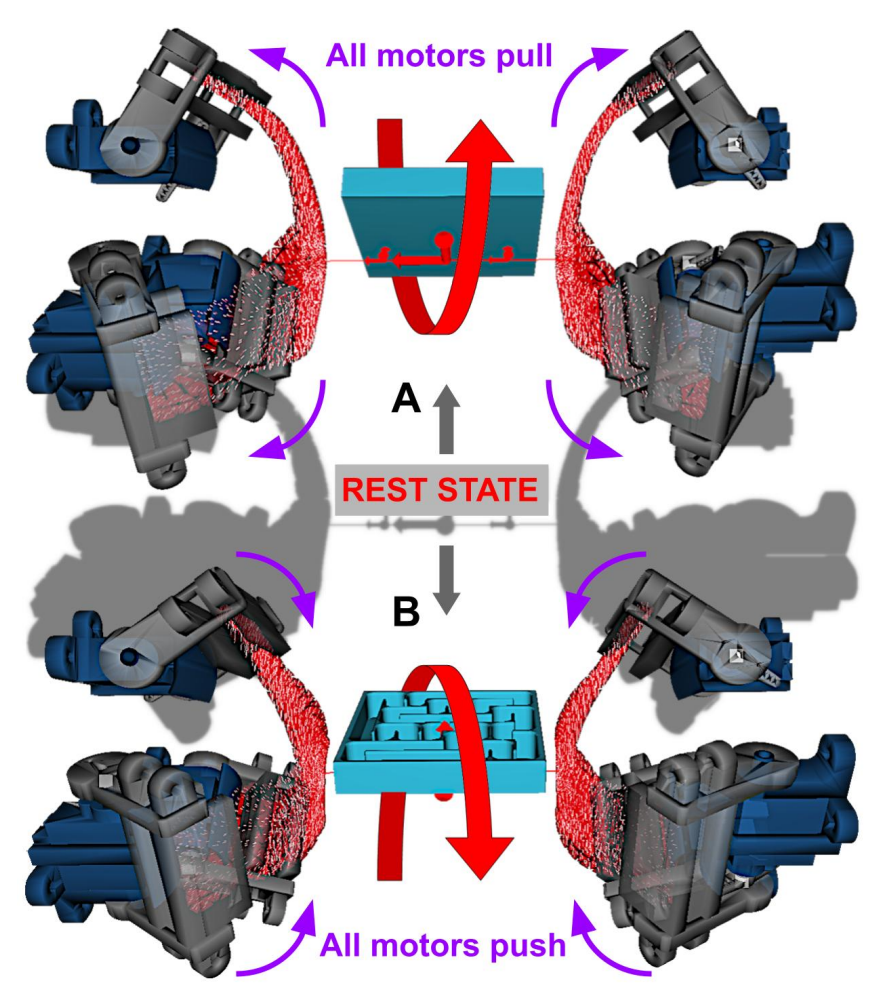

Fig. 4: Side view of the simulation of the global setup. In the middle, in grey, the system is presented at rest state. The figure show the sixth DOF around the rod. When both tripod "pull" (A), a rotation appears around the rod in one direction and when they "push" (B) it rotate on the same axis but in an opposite direction. Due to the fact that motors are either all "pulling" or all "pushing", the maze stay centered during all the actuation enabling a decoupled sixth DOF

\section{FEM SIMULATION}

In this section we will first present how anisotropic soft robots are simulated using non-linear FEM and how FEM is coupled with the rigid link and the rigid actuators. Then, we compute the Jacobian of the robot direct kinematics. Finally, we provide the optimisation used to obtain the inverse model of the robot.

\section{A. Finite Element Model of Anisotropic Soft Robots}

For the FEM, we rely on the framework SOFA 1 At static equilibrium the external and internal forces are in equilibrium for each node of the mesh:

$$
\mathbf{0}=\mathbf{M g}-\mathbf{f}(\mathbf{x})+\tilde{\mathbf{J}}_{\mathbf{a}}^{T} \boldsymbol{\lambda}
$$

where $\mathrm{x}$ is the vector of position of the FEM nodes, $\mathbf{M}$ is the mass matrix (we use mass lumping), and $\mathbf{g}$ the gravity exerted on each node, $\mathbf{f}$ accounts for internal forces (depending on the material the soft robots is made of), and $\tilde{\mathbf{J}}_{\mathrm{a}}^{T} \boldsymbol{\lambda}$ is the vector of constraint forces contributions which are generated when an actuator applies force on the soft

\footnotetext{
${ }^{1}$ wwww.sofa-framework.org
}

robot [12]. In the particular case of our robot the actuation is provided by an articulated joint, so $\tilde{\mathbf{J}}_{\mathbf{a}}$ is similar to a pseudo inverse of the Jacobian for an articulated rigid system (see below for details).

Mechanical metamaterials induce new or different behaviors and responses to stimuli that differ from normal isotropic material (e.g: homogeneous material having the same mechanical properties along each axis), we call that phenomenon anisotropy. In such case, the material has a characteristic orientation.

In practice, the anisotropy will affect the computation of the internal forces $\mathbf{f}(\mathbf{x})$ in the FEM. we use a special case of anisotropy: transverse isotropic. This kind of anisotropy implies that the solid can be rotated with respect to the loading direction about one axis without measurable effect on the solid's response. It leads to the definition of 5 mechanical parameters, which are sufficient to describe correctly the deformation of the metamaterial. The parameters are : a transversal $\left(\boldsymbol{E}_{t}\right)$ and longitudinal $\left(\boldsymbol{E}_{l}\right)$ Young's moduli, a transversal poisson ratio $\left(\nu_{t}\right)$, a transversal longitudinal poisson ratio $\left(\nu_{t l}\right)$ and finally the longitudinal shear modulus $\left(\mu_{l}\right)$. We obtain the values of those mechanical parameters by using homogenization (for more details see [10]).

To reproduce the anisotric behavior of the 3D printed flexible sheet, we give a specific orientation of each elements of our mesh in order to produce a spiral pattern as shown on Fig. 3. We can "play" with the values $\boldsymbol{E}_{\boldsymbol{t}}$ and $\boldsymbol{E}_{\boldsymbol{l}}$ to have a material more flexible/rigid along $\boldsymbol{E}_{t}$ direction or $\boldsymbol{E}_{l}$ direction.

\section{B. Rigid coupling}

Let's name $q_{e}$ the vector containing the 6 DOF rigid positions of the effector and $q_{a}$ the 1 DOF rigid articulation of the actuators. Some of the 3 DOF nodes of the meshes are attached to these rigid bodies and are kinematically linked to their rigid position. So if we split the position vector of the nodes in three parts: $\mathbf{x}_{\mathbf{e}}$ the nodes that are rigidified on the effector body, $\mathrm{x}_{\mathrm{a}}$ that are rigidified on the actuator body and $\mathbf{x}_{\mathbf{f}}$ the nodes that are kept free to deform. We obtain that the position $\mathbf{x}$ of the mesh nodes depends on $\mathbf{q}_{e}, \mathbf{q}_{a}$ and $\mathbf{x}_{\mathbf{f}}$ : $\mathbf{x}=\mathbf{x}\left(\mathbf{q}_{\mathbf{e}}, \mathbf{q}_{\mathbf{a}}, \mathbf{x}_{\mathbf{f}}\right)$. Let's now compute the derivatives:

$$
d \mathbf{x}=\left[\begin{array}{l}
\mathbf{J}_{\mathbf{e}}\left(\mathbf{q}_{\mathbf{e}}\right) d \mathbf{q}_{\mathbf{e}} \\
\mathbf{J}_{\mathbf{a}}\left(\mathbf{q}_{\mathbf{a}}\right) d \mathbf{q}_{\mathbf{a}} \\
d \mathbf{x}_{\mathbf{f}}
\end{array}\right]
$$

with $\mathbf{J}_{\mathbf{e}}=\frac{\partial \mathbf{x}\left(\mathbf{q}_{\mathbf{e}}, \mathbf{q}_{\mathbf{a}}, \mathbf{x}_{\mathbf{f}}\right)}{\partial \mathbf{q}_{\mathbf{e}}}$ and $\mathbf{J}_{\mathbf{a}}=\frac{\partial \mathbf{x}\left(\mathbf{q}_{\mathbf{e}}, \mathbf{q}_{\mathbf{a}}, \mathbf{x}_{\mathbf{f}}\right)}{\partial \mathbf{q}_{\mathbf{a}}}$ the Jacobian matrices that are classically used for rigid bodies in robotics. The transposed Jacobians $\mathbf{J}_{\mathbf{e}}{ }^{T}$ and $\mathbf{J}_{\mathbf{a}}{ }^{T}$ are used to bring the internal forces exerted on the rigidified nodes into torques and forces of their rigid body.

To study the kinematics, let's take a case where we slightly change the actuation force $\boldsymbol{\lambda}$ and change it by $\boldsymbol{\lambda}+d \boldsymbol{\lambda}$. It will create a small motion $d \mathbf{x}$ of the FEM mesh nodes. Let's consider the difference between the two static equilibrium described in equation (1) taken at position $\mathbf{x}+d \mathbf{x}$ and at 
position $\mathbf{x}$ :

$$
\mathbf{f}(\mathbf{x}+d \mathbf{x})-\mathbf{f}(\mathbf{x})) \approx \underbrace{\frac{\partial \mathbf{f}(\mathbf{x})}{\partial \mathbf{x}}}_{\mathbf{K}(\mathbf{x})} d \mathbf{x}=\tilde{\mathbf{J}}_{\mathbf{a}}^{T} d \boldsymbol{\lambda}
$$

with $\mathbf{K}(\mathbf{x})$ the non-linear tangent of the internal forces (often called the stiffness matrix). We can project the forces into effector and actuator spaces (where lambda, unknown joint torque, is defined and removes the pseudo-inverse $\tilde{\mathbf{J}}_{\mathbf{a}}^{T}$ ):

$$
\begin{aligned}
& \mathbf{J}_{\mathbf{e}}^{T} \mathbf{K}(\mathbf{x}) d \mathbf{x}=0 \\
& \mathbf{J}_{\mathbf{a}}^{T} \mathbf{K}(\mathbf{x}) d \mathbf{x}=d \boldsymbol{\lambda}
\end{aligned}
$$

The size of this matrix is 3 times the number of nodes of the FEM (as each node has 3 DOF). Let's consider that we can extract blocks $\mathbf{K}_{\mathbf{i j}}$ on this matrix where $\mathbf{i}$ and $\mathbf{j}$ can be the set of nodes $\mathbf{e}, \mathbf{a}$ or $\mathbf{f}$. If we combine (5) with (2) we have :

$$
\underbrace{\left[\begin{array}{lll}
\mathbf{J}_{\mathbf{e}}{ }^{T} \mathbf{K}_{\mathbf{e e}} \mathbf{J}_{\mathbf{e}} & \mathbf{J}_{\mathbf{e}}{ }^{T} \mathbf{K}_{\mathbf{e a}} \mathbf{J}_{\mathbf{a}} & \mathbf{J}_{\mathbf{e}}{ }^{T} \mathbf{K}_{\mathbf{e f}} \\
\mathbf{J}_{\mathbf{a}}{ }^{T} \mathbf{K}_{\mathbf{a e}} \mathbf{J}_{\mathbf{e}} & \mathbf{J}_{\mathbf{a}}{ }^{T} \mathbf{K}_{\mathbf{a a}} \mathbf{J}_{\mathbf{a}} & \mathbf{J}_{\mathbf{a}}{ }^{T} \mathbf{K}_{\mathbf{a f}} \\
\mathbf{K}_{\mathbf{f e}} \mathbf{J}_{\mathbf{e}} & \mathbf{K}_{\mathbf{f a}} \mathbf{J}_{\mathbf{a}} & \mathbf{K}_{\mathbf{f f}}
\end{array}\right]}_{\mathbf{A}}\left[\begin{array}{l}
d \mathbf{q}_{\mathbf{e}} \\
d \mathbf{q}_{\mathbf{a}} \\
d \mathbf{x}_{\mathbf{f}}
\end{array}\right]=\left[\begin{array}{l}
\mathbf{0} \\
d \boldsymbol{\lambda} \\
\mathbf{0}
\end{array}\right]
$$

This system can be solved to compute the motion of the robot due to the change of forces in the actuation and in particular we can get the direct Jacobian between actuators and effector by using some blocks of the inverse matrix $\mathbf{A}^{-1}=\mathbf{W}$, which is the compliance matrix :

$$
d \mathbf{q}_{\mathbf{e}}=\mathbf{W}_{\mathbf{e a}}\left[\mathbf{W}_{\mathbf{a a}}\right]^{-1} d \mathbf{q}_{a}
$$

The form of this kinematic model of soft robot is generic. This is the same as in [13].

To obtain the inverse kinematics, we can simply inverse the equation (7) but in practice we also need to define some limits in the actuation (in particular the course of the actuators). So we use quadratic programming in order to minimize the quadratic distance $\frac{1}{2} d \mathbf{q}_{\mathbf{e}}{ }^{T} d \mathbf{q}_{\mathbf{e}}$ between the current effector position $\mathbf{q}_{e}$ and the goal position $\mathbf{q}_{e}^{*}$ :

$$
\left\{\begin{array}{l}
\min \left(\frac{1}{2} d \boldsymbol{\lambda}^{T} \mathbf{W}_{\mathbf{e a}}^{T} \mathbf{W}_{\mathbf{e a}} d \boldsymbol{\lambda}^{T}+d \boldsymbol{\lambda}^{T} \mathbf{W}_{\mathbf{e a}}^{T} d \mathbf{q}_{\mathbf{e}}{ }^{0}\right) \\
\text { subject to : (course of the actuators }) \\
d \mathbf{q}_{\mathbf{a}}{ }^{\text {min }} \leq \mathbf{W}_{\mathbf{a a}} d \boldsymbol{\lambda}+d \mathbf{q}_{\mathbf{a}}{ }^{0} \leq d \mathbf{q}_{\mathbf{a}}{ }^{\max }
\end{array}\right.
$$

\section{WORKSPACE EVALUATION}

In this section we present how we perform a workspace exploration using the model presented above and provide a discussion about the results. We also present an evaluation of the anisotropy influence on the 6th DOF.

\section{A. Method of evaluation}

The workspace exploration is done in a discrete way using the inverse problem presented in eq (8). We constrain the motors with maximum and minimum possible values of their shaft in order to have a work space of 90 degree in total for each motor, with 0 degree having the motor totally "horizontal" and 90 degree "vertical".

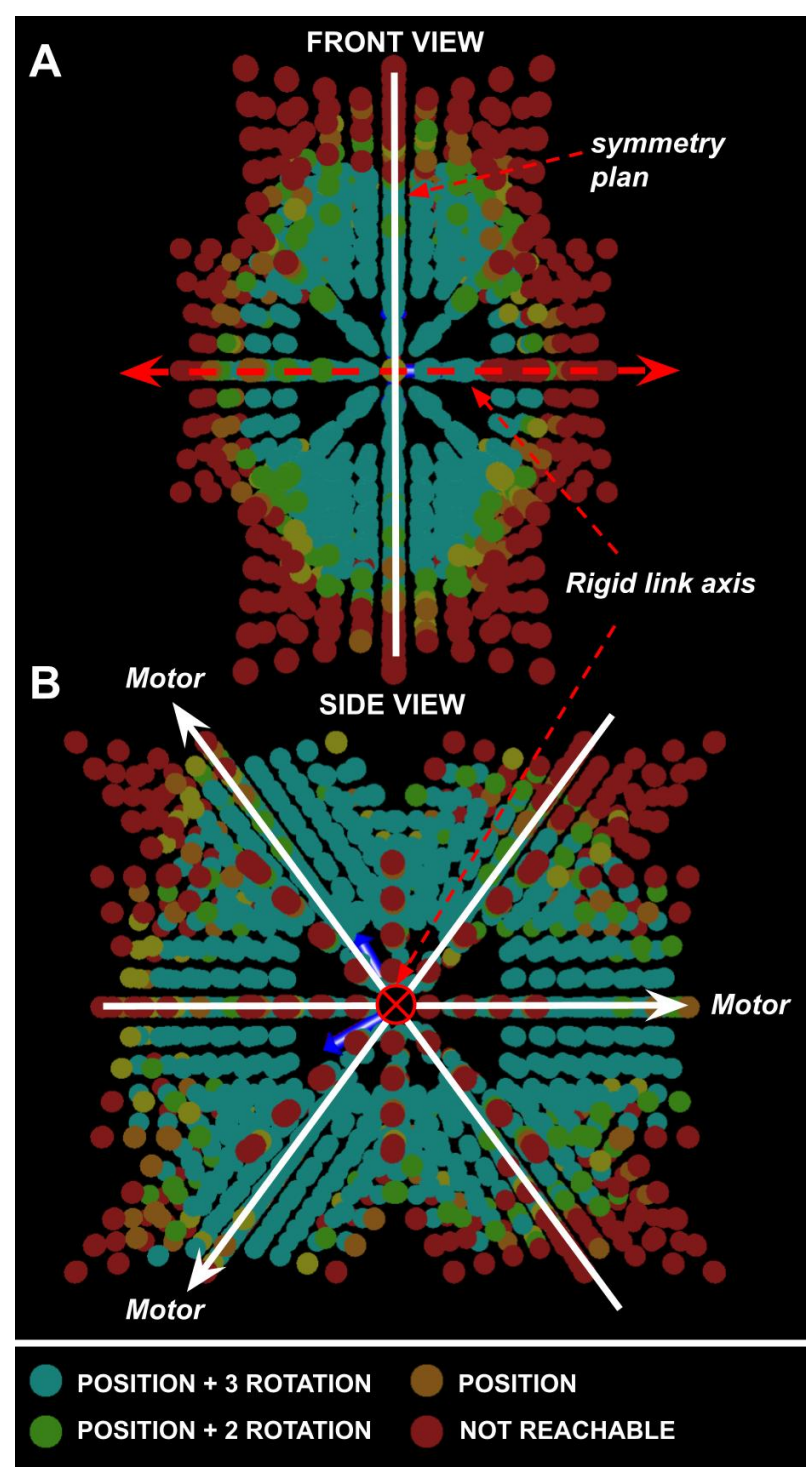

Fig. 5: Workspace exploration with the effector. Starting from rest position, we try to reach each point and if it is reachable we try to do on it 3 orthogonal rotation of $-/+10$ degrees. Depending on the results the point is classified in the blue, green, orange or red categories.

We generate a grid of point along the directions of actuation, this produce a kind of 3D star workspace to explore. In total, we have around 1800 points to explore. we choose to remove most of points around the center in order to gain time during the workspace exploration. We then begin the evaluation by moving the goal from point to point and verifying with a error margin of $1 \mathrm{~mm}$ if the inverse model succeeds to reach it. We then do the same process again but adding an evaluation of the rotations capability on each point of the workspace. We apply successively 3 rotations (along $\mathrm{x}, \mathrm{y}$ and $\mathrm{z}$ ) with minus or plus 10 degrees and with an error margin of 2 degrees. In fonction of the rotation results we create 3 categories in which the point will be classified : points on which we can do the 3 rotations, points on which 
we can only do 2 out of the 3 rotations and finally points which are only reachable but on which we can not do any rotation.

Of course, the workspace is influenced by many different parameters. Some of them are easy to set in the inverse problem, like the angular boundaries of the motors. In a last workspace exploration we show its influence as we do multiple exploration, each time with different angular boundaries of our actuators in order to see its influence on the final workspace.

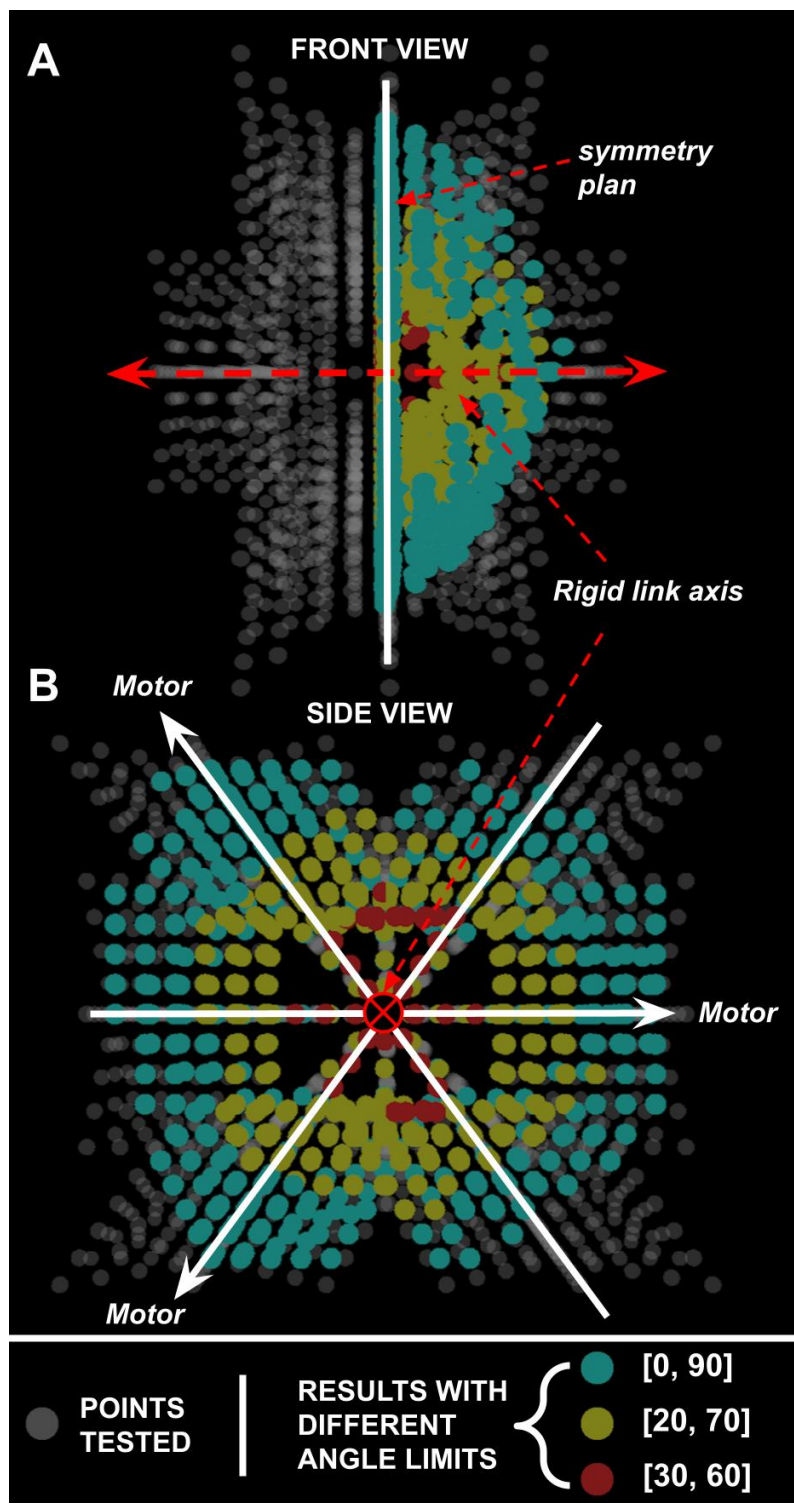

Fig. 6: Workspace exploration with different angle limits imposed on the actuators. In this constrained exploration, reaching a points with an error margin of $1 \mathrm{~mm}$ validate it. The exploration is done on the same points of Fig. 5. We test 3 different angle limits producing 3 different possible workspace each including the other : red is included in yellow which is included in blue.

\section{B. Results and interpretation}

The workspace exploration is shown on Fig. 5

We observe that at the initial position (center of workspace) and in a whole area around it, we have a full 6 DOF control of the end-effector. Then, as we translate the effector further to the side, we gradually lose the control of the different rotations up to the positions on the boundary of the workspace where we are only able to perform translations.

Perpendicular to the rigid link we observe in the front view (A) that the workspace is symmetrical which is expected due to the physical symmetry of the system.Another remark is that the final workspace has a star shape which can be explained by the fact there is an asymmetry between the points lying directly in the direction of the motors axis and other points lying in the directions passing in between two motors.

In Fig. 6, we can see the superposition of different workspaces produced by changing successively the possible range of actuation of the motors. As could be expected, as the range decreases, the workspace reduces towards the center.

We noted that the exploration of the workspace is sometimes not realistic: for instance, when increasing the rotation level from 10 degrees to 20 degrees and performing again the exploration shown in Fig 5 we would expect a reduction of the workspace. But, in such case, the working space is only very (too) slightly reduced. This is probably due to an idealization of the constitutive law in the FEM model (see the Discussion section).

\section{Anisotropy influence}

A new experiment was conducted to demonstrate more clearly the influence of the anisotropy on the kinematics. To this end, we have two extreme cases: on one hand, the isotropic case and on the other hand the anisotropy case used for the final design of the system. To create intermediate cases, we interpolate between this two extreme cases. The Fig. 8 demonstrates that the rotation of the end-effector appears progressively for the same input motion on the motors.

\section{MODEL VALIDATION}

In this section we present how the real robot was made and then compare its behavior with its intended design previously simulated and show its maneuverability by using it to solve a maze.

\section{A. Real Model Construction}

For the fabrication of the metamaterial composing our flexible sheet and have something close to the one presented in Fig. 3. we are using fused filament fabrication (FFF) printing technique and an existing method for programming stiffness through stochastic foam. This method comes from the works of [11] and is implemented in a special slicer called IceSL This slicer give us the ability to "paint" on a given shape a desired flexibility and the directions in which

${ }^{2}$ IceSL: https://icesl.loria.fr 


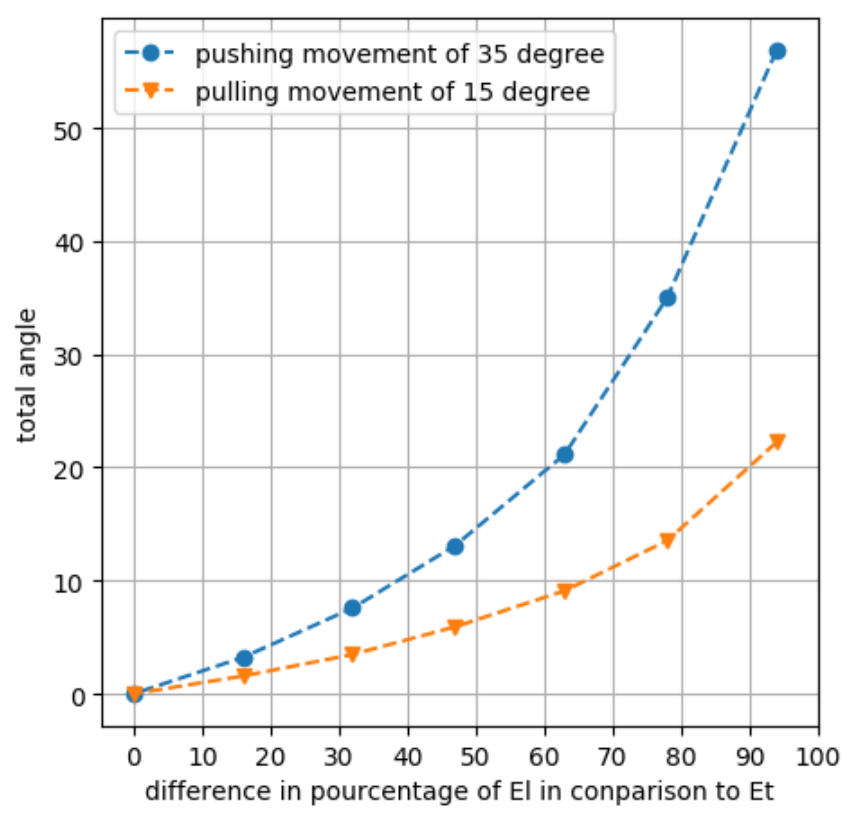

Fig. 7: Convergence from transverse (right of the figure) to isotropic (origin). Abscissa $\alpha$ represents an anisotropy percentage. $\alpha(\%)=100-100\left(E_{l} / E_{t}\right)$. The test is done by either pulling all motors of 15 degrees or pushing them of 35 degrees from the rest state as presented in Fig. 4

the densities are increased or reduced. We can see in Fig. 3 superimposed to the simulated part the real one produced by IceSL.

In practice, the soft structures used for this paper are 3D printed with a basic desktop 3D printer (Prusa i3 MKS) with a thermoplastic polyurethane (TPU) called NinjaFlex (Shore Hardness: $85 \mathrm{~A}$ and a tensile modulus of $12 \mathrm{MPa}$ : data from the constructor) with $1.75 \mathrm{~mm}$ of diameter. We print with a layer thickness of $0.3 \mathrm{~mm}$ and a print speed of $25 \mathrm{~mm} / \mathrm{sec}$. We choose this TPU in particular because it is inherently compliant. It is one of the few commercially available filament for FFF 3D printing with low shore hardness and, printed as a stochastic foam, we obtain great deformations without having to put to much load.

We mount the flexible structure produced on the tripod and then link them together with a metal rod on which we attach on the middle a 3D printed (with traditional FFF technique using PLA) maze. This system is afterwards mounted into a rigid box allowing the whole system to stay stable when the two tripods actuate against each other. During the mounting process we put particular attention in keeping the same distance as in the simulation. We can see in Fig. 2 the whole final setup at rest state.

\section{B. Validation}

Here, we are looking for the three following properties :

- A new independent DOF is created and controllable.

- We can still control independently the 5 other DOF.

- The numerical model is sufficiently accurate to control the robot in open loop.
1) Additional Independent Controllable DOF: We can see in Fig. 8 for the same actuation the different results for a parallel soft robot with sheets made of either silicone or metamaterial. We can confirm that with silicone no central rotation is created but rather that one of the tripod sheet buckle due to the pression of the other resulting in a lateral translation. For the robot made with sheet composed of metamaterial, we have, as expected from the simulation, the creation of a rotation around the rigid axis, while creating no translation. This demonstrates the possibility of controlling a new independent DOF. The rotation the other way around when all the motors are pulling is also verified.

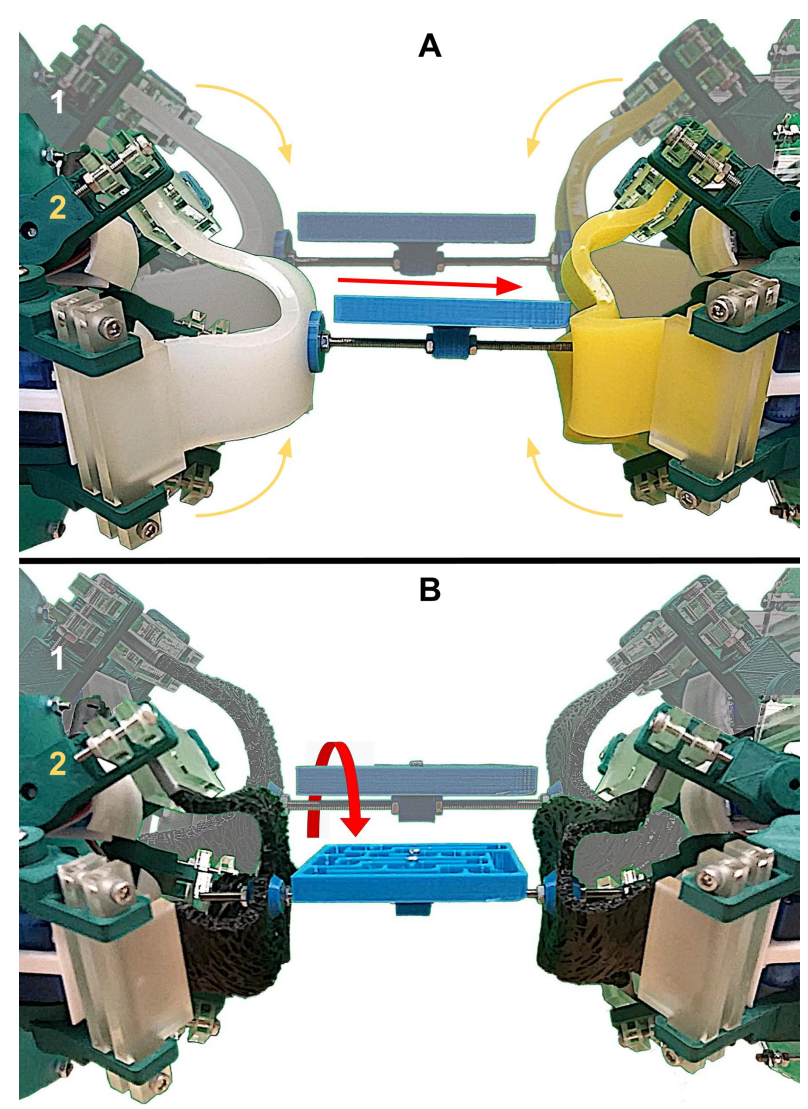

Fig. 8: Comparison between a parallel soft robot made of silicon (A) and one made of our metamaterial (B). For both we actuate all servomotors from 1 to 2; action : yellow arrow / reaction : red arrow. With traditional homogeneous material (A), we can see that the maze translate to the right, but with a mesostructured flexible sheet $(\mathbf{B})$ a rotation around the metal rod axes is created.

2) Keeping the independent control of the 5 other DOF: Now that we have successfully managed to get control of the rotation of the rod, we make sure all remaining 5 DOF are controllable independently, i.e we can still translate the effector without parasitic rotations for example. It is the case indeed, and it can be seen in the video material attached with this paper. 
3) Maze Solving: We put a marble on the maze and we were able to impose the motion of the effector in the global frame, in order to solve it. What we mean by solving it, is that we were able, in one go, to make the marble follow the whole path of the maze and come back at the initial position by actuating both tripod and controlling the different inclinations of the maze demonstrating in doing so its maneuverability and controllability. In particular, to solve the maze, we need to use the newly created additional DOF created with the anisotropic material and cannot solve it using a setup built with silicone.

\section{DISCUSSION}

Overall, the results demonstrate the possibility of controlling a new degree of freedom on a parallel soft robot using anisotropic material. We also show that using the modeling approach, and in particular the inverse model, the motions of the end-effector can be decoupled and controlled independently in open-loop.

This modeling approach is also used to evaluate the workspace of the robot. But here, we would like to emphasize that this evaluation is discrete and does not prove that all trajectories are possible inside this workspace ! In particular, in some positions, one of the soft sheet legs is buckling and the corresponding motor can only poorly affect the motion of the effector, meaning that we probably lose control of the 6 DOF. Consequently, there is probably much more to study concerning the workspace analysis and what is presented here is only a method for first evaluation.

An other limitation concerns the homogenization. This is inherently a simplification of the behavior of the stochastic foam. In particular self-collisions inside the structure due to large strain are not taken into account. So, in practice, the FEM model is much less constrained geometrically when some local strain are large. This leads to an overestimation of the workspace of the model compared to the reality. We probably have to reproduce the effect of these self-collisions, or add a criterion to limit the maximum strain, or at least evaluate the domain of validity of the homogenization.

\section{CONCLUSIONS AND PERSPECTIVES}

This paper opens up a new perspectives in the design of parallel soft robots. We demonstrate that the designer can play with anisotropy to control (or improve the control) of degrees of freedom. This paper also shows that numerical approaches can be developed to help the design of these robots whose kinematics are influenced by the constitutive law of the materials. In one hand, we can program more complex materials than silicone (such as stochastic foams). On the other hand, we can also have a first analysis of the workspace thanks to FEM. But this paper also highlights the amount of work that remains to be done: to have proofs of continuity in the workspace, to better model the small-scale behavior and in particular the collisions in the material, to control the dynamics of such robot, and finally to see in which application cases these parallel robots, that are simple to manufacture, are more suitable than rigid parallel robots.

\section{ACKNOWLEDGMENTS}

We thank Sylvain Lefebvre and Jonàs Martínez for their help with the software IceSL and mesostructure homogenization. We also thank Hervé Delingette for his initial work on the framework SOFA for simulating anisotropic structure. This work was supported by INRIA and le Conseil Région Hauts de France.

\section{REFERENCES}

[1] Robert F Shepherd et al. "Multigait soft robot". In: Proceedings of the national academy of sciences 108.51 (2011), pp. 20400-20403.

[2] Matteo Cianchetti et al. "Soft robotics technologies to address shortcomings in today's minimally invasive surgery: the STIFF-FLOP approach". In: Soft robotics 1.2 (2014), pp. 122-131.

[3] Marcello Calisti et al. "An octopus-bioinspired solution to movement and manipulation for soft robots". In: Bioinspiration \& biomimetics 6.3 (2011), p. 036002.

[4] Steven A Cummer, Johan Christensen, and Andrea Alù. "Controlling sound with acoustic metamaterials". In: Nature Reviews Materials 1.3 (2016), pp. 1-13.

[5] Katia Bertoldi et al. "Flexible mechanical metamaterials”. In: Nat. Rev. Mater. 2 (2017).

[6] Ahmad Rafsanjani, Katia Bertoldi, and André R. Studart. "Programming soft robots with flexible mechanical metamaterials". In: Sci. Robot. 4.29 (2019).

[7] Jakob A Faber, Andres F Arrieta, and André R Studart. "Bioinspired spring origami". In: Science 359.6382 (2018), pp. 1386-1391.

[8] Tian Chen et al. "Harnessing bistability for directional propulsion of soft, untethered robots". In: Proceedings of the National Academy of Sciences 115.22 (2018), pp. 5698-5702.

[9] Melanie F Simons et al. "Tiled Auxetic Cylinders for Soft Robots". In: 2019 RoboSoft. IEEE. 2019, pp. 6267.

[10] Félix Vanneste et al. "Anisotropic soft robots based on 3D printed meso-structured materials: design, modeling by homogenization and simulation". In: IEEE Robotics and Automation Letters 5.2 (2020), pp. 2380-2386.

[11] Jonàs Martınez et al. "Polyhedral Voronoi diagrams for additive manufacturing". In: ACM Transactions on Graphics (TOG) 37.4 (2018), p. 129.

[12] Eulalie Coevoet et al. "Software toolkit for modeling, simulation, and control of soft robots". In: Advanced Robotics 31.22 (2017), pp. 1208-1224.

[13] Thor Morales Bieze et al. "Finite element methodbased kinematics and closed-loop control of soft, continuum manipulators". In: Soft robotics 5.3 (2018), pp. 348-364. 